\title{
A New Method Based on the RKHSM for Solving Systems of Nonlinear IDDEs with Proportional Delays
}

\author{
Changbo He, ${ }^{1}$ Xueqin $\mathrm{Lv}^{2}$ and Jing $\mathrm{Niu}^{2}$ \\ ${ }^{1}$ School of Sports and Sciences, Harbin Normal University, Harbin, Heilongjiang 150025, China \\ ${ }^{2}$ School of Mathematics and Sciences, Harbin Normal University, Harbin, Heilongjiang 150025, China \\ Correspondence should be addressed to Xueqin Lv; hashidalvxueqin@126.com
}

Received 10 November 2012; Revised 1 March 2013; Accepted 25 March 2013

Academic Editor: Zhenya Yan

Copyright (c) 2013 Changbo He et al. This is an open access article distributed under the Creative Commons Attribution License, which permits unrestricted use, distribution, and reproduction in any medium, provided the original work is properly cited.

An efficient computational method is given in order to solve the systems of nonlinear infinite-delay-differential equations (IDDEs) with proportional delays. Representation of the solution and an iterative method are established in the reproducing kernel space. Some examples are displayed to demonstrate the computation efficiency of the method.

\section{Introduction}

In functional-differential equations (FDEs), there is a class of infinite-delay-differential equations (IDDEs) with proportional delays such systems are often encountered in many scientific fields such as electric mechanics, quantum mechanics, and optics. In view of this, developing the research for this class of IDDEs possesses great significance on theory and practice, for this attracts constant interest of researchers. Ones have found that there exist very different mathematical challenges between FDEs with proportional delays and those with constant delays. Some researches on the numerical solutions and the corresponding analysis for the linear FDEs with proportional delays have been presented by several authors. In the last few years, there has been a growing interest in studying the existence of solutions of functional differential equations with state dependent delay [1-7]. Initial-value problem for neutral functional-differential equations with proportional time delays had been studied in [8-11]; in the literature [11] authors had discussed the existence and uniqueness of analytic solution of linear proportional delays equations.

Ishiwata et al. used the rational approximation method and the collocation method to compute numerical solutions of delay-differential equations with proportional delays in $[12,13]$. At [14-17], Hu et al. gave the numerical method to compute numerical solutions of neutral delay differential equations. For neutral delay differential equations with proportional delays, Chen and Wang proposed the variational iteration method [18] and the homotopy perturbation method [19]. Recently, time-delay systems become interested in applications like population growth models, transportation, communications, and agricultural models so those systems were widely studied both in a theoretical aspect and in that of related applications [20-22].

We consider the following nonlinear infinite delaydifferential equation (IDDE) with proportional delay [23]:

$$
\begin{gathered}
u^{\prime}(x)=g(x, u(x), u(p x)), \\
u(0)=\eta,
\end{gathered}
$$

where $p \in(0,1), \eta$ is a given initial value, $u(x) \in W_{2}[0,+\infty)$, and for $x \in[0,+\infty), y, z \in(-\infty,+\infty), g(x, y, z)$ is a continuous function; $g(x, y, z) \in W_{1}[0,+\infty)$ as $y=y(x)$, $z=z(x) \in W_{1}[0,+\infty)$.

Next, the following system of nonlinear infinite-delaydifferential equations (IDDEs) with proportional delays will be studied:

$$
\begin{aligned}
& u^{\prime}(x)=F(x, u(x), v(p x)), \\
& v^{\prime}(x)=G(x, v(x), u(q x)), \\
& u(0)=0, \quad v(0)=0,
\end{aligned}
$$

where $p, q \in(0,1)$, for $x \in[0,+\infty), y, z \in(-\infty,+\infty)$, $f(x, y, z), g(x, y, z)$ are continuous bounded function, and 
$f(x, y, z) \in W_{1}[0,+\infty), g(x, y, z) \in W_{1}[0,+\infty)$ as $y=y(x)$, $z=z(x) . W_{2}[0,+\infty)$ and $W_{1}[0, \infty)$ are reproducing kernel spaces. Equations (2) are obtained through homogenization of initial condition for model in [24]. In this study, existence and a new iterative algorithm are established for the nonlinear infinite-delay-differential equations (IDDEs) with proportional delays in the reproducing kernel spaces.

The paper is organized as follows. In Section 2, some definitions of the reproducing kernel spaces are introduced. In Section 3, main results and the structure of the solution for operator equation are discussed. Existence of the solution to (2) and an iterative method are developed for the kind of problems in the reproducing kernel space. We verify that the approximate solution converges to the exact solution uniformly. In Section 4, some experiments are given to demonstrate the computation efficiency of the algorithm. The conclusion is given in Section 5.

\section{Preliminaries}

Definition 1 (see [25] (reproducing kernel)). Let $M$ be a nonempty abstract set. A function $K: M \times M \rightarrow C$ is a reproducing kernel of the Hilbert space $H$ if and only if

(a) $\forall y \in M, K(\cdot, x) \in H$,

(b) $\forall y \in M, \forall u \in H,\langle u(\cdot), K(\cdot, y)\rangle=u(y)$.

The condition (b) is called "the reproducing property"; a Hilbert space which possesses a reproducing kernel is called a Reproducing Kernel Hilbert Space (RKHS).

Next, two reproducing kernel spaces are given.

Definition 2. $W_{2}[0,+\infty)=\left\{u(x) \mid u^{\prime}\right.$ is absolutely continuous real value functions, $\left.u^{\prime \prime} \in L^{2}[0,+\infty), \quad u(0)=0\right\}$.

$W_{2}[0,+\infty)$ is a Hilbert space, for $u(x), v(x) \in W_{2}[0,+\infty)$; the inner product and norm in $W_{2}[0,+\infty)$ are given by

$$
\begin{gathered}
\langle u(x), v(x)\rangle_{W_{2}}=\int_{0}^{+\infty}\left(4 u v+5 u^{\prime} v^{\prime}+u^{\prime \prime} v^{\prime \prime}\right) d x, \\
\|u\|_{W_{2}}=\sqrt{\langle u, u\rangle_{W_{2}}},
\end{gathered}
$$

respectively.

Theorem 3. The space $W_{2}[0,+\infty)$ is a reproducing kernel space that is, for any $u(y) \in W_{2}[0,+\infty)$ and each fixed $x \in$ $[0,+\infty)$, there exists $R_{x}(y) \in W_{2}[0,+\infty), y \in[0,+\infty)$, such that $u(x)=\left\langle R_{x}(y), u(y)\right\rangle$. And the corresponding reproducing kernel can be represented as follows [26]:

$$
R_{x}(y)= \begin{cases}-\frac{1}{12} e^{-2(x+y)}\left(-1+e^{2 y}\right)\left(1+e^{2 y}-2 e^{x+y}\right), & y \leq x, \\ -\frac{1}{12} e^{-2(x+y)}\left(-1+e^{2 x}\right)\left(1+e^{2 x}-2 e^{x+y}\right), & y>x .\end{cases}
$$

Definition 4. $W_{1}[0,+\infty)=\{u(x) \mid u$ is absolutely continuous real-valued function, $\left.u^{\prime} \in L^{2}[0,+\infty)\right\}$. by

The inner product and norm in $W_{1}[0,+\infty)$ can be defined

$$
\begin{aligned}
\langle u(x), v(x)\rangle_{W_{1}} & =\int_{0}^{+\infty}\left(u v+u^{\prime} v^{\prime}\right) d x, \\
\|u\|_{W_{1}} & =\sqrt{\langle u, u\rangle_{W_{1}}},
\end{aligned}
$$

respectively, where $u(x), v(x) \in W_{1}[0,+\infty)$. It has been proved that $W_{1}[0,+\infty)$ is a complete reproducing kernel space and its reproducing kernel is as follows [27]:

$$
Q_{x}(y)= \begin{cases}\frac{1}{2} e^{-x-y}\left(1+e^{2 x}\right), & x<y \\ \frac{1}{2} e^{-x-y}\left(1+e^{2 y}\right), & x \geq y .\end{cases}
$$

\section{Statements of the Main Results}

In this section, the implementation method of obtaining the solution of (2) is proposed in the reproducing kernel space $W_{2}[0,+\infty)$.

Put the differential operator $\mathscr{L}=d / d x$; then we can convert (2) into the following form:

$$
\begin{aligned}
& \mathscr{L} u(x)=F(x, u(x), v(p x)), \\
& \mathscr{L} v(x)=G(x, v(x), u(q x)), \\
& u(0)=v(0)=0,
\end{aligned}
$$

where $x \in[0,+\infty)$, and

$$
\begin{gathered}
F(x, u(x), v(p x)) \stackrel{\text { def }}{=} N(u(x), v(p x))+f(x), \\
G(x, v(x), u(q x)) \stackrel{\text { def }}{=} D(v(x), u(q x))+g(x) .
\end{gathered}
$$

It is clear that $\mathscr{L}: W_{2}[0,+\infty) \rightarrow W_{1}[0,+\infty)$ is a bounded linear operator. Let $\varphi_{i}(x)=Q_{x_{i}}(x)$, where $\left\{x_{i}\right\}_{i=1}^{\infty}$ is dense in the interval $[0,+\infty)$, and $\psi_{i}(x)=\mathscr{L}^{*} \varphi_{i}(x)$, where $\mathscr{L}^{*}$ is the conjugate operator of $\mathscr{L}$. Define the normal orthogonal system $\bar{\psi}_{i}(x)$ in $W_{2}[0,+\infty)$, which derives from Gram-Schmidt orthogonalization process of $\left\{\psi_{i}(x)\right\}_{i=1}^{\infty}$,

$$
\overline{\psi_{i}}(x)=\sum_{k=1}^{i} \beta_{i k} \psi_{k}(x), \quad \beta_{i i}>0, \quad i=1,2, \ldots
$$

Lemma 5. Assume that $\left\{x_{i}\right\}_{i=1}^{\infty}$ are dense in $[0,+\infty)$; then $\left\{\psi_{i}(x)\right\}_{i=1}^{\infty}$ is a complete system in $W_{2}[0,+\infty)$ and $\left\{\psi_{i}(x)\right\}=$ $\left.(d / d y) R_{x}(y)\right|_{y=x_{i}}$.

Proof. Ones have

$$
\begin{aligned}
\psi_{i}(x) & =\left\langle\psi_{i}(y), R_{x}(y)\right\rangle_{W_{2}}=\left\langle\mathscr{L}^{*} \varphi_{i}(y), R_{x}(y)\right\rangle_{W_{2}} \\
& =\left\langle\varphi_{i}(y), \mathscr{L} R_{x}(y)\right\rangle_{W_{1}}=\mathscr{L} R_{x}\left(x_{i}\right) \\
& =\left.\frac{d}{d y} R_{x}(y)\right|_{y=x_{i}} .
\end{aligned}
$$

Clearly, $\psi_{i}(x) \in W_{2}[0,+\infty)$. 
For any $u(x) \in W_{2}[0,+\infty)$, let $\left\langle u(x), \psi_{i}(x)\right\rangle=0, i=$ $1,2, \ldots$, which means that

$$
\left\langle u(x),\left(\mathscr{L}^{*} \varphi_{i}(x)\right\rangle=\left\langle\mathscr{L} u(\cdot), \varphi_{i}(\cdot)\right\rangle=(\mathscr{L} u)\left(x_{i}\right)=0 .\right.
$$

Note that $\left\{x_{i}\right\}_{i=1}^{\infty}$ is dense in $[0,+\infty)$; therefore, $\mathscr{L} u(x)=0$. It follows that $u(x) \equiv 0$ from the existence of $\mathscr{L}^{-1}$.

3.1. Construction the of Iterative Sequence $\tilde{u}_{n}(x)$ and $\widetilde{v}_{n}(x)$. Next we construct the iterative sequence $\tilde{u}_{n}(x)$ and $\widetilde{v}_{n}(x)$, putting

$$
\begin{gathered}
\mathscr{L} M_{n}(x)=F\left(x, \tilde{u}_{n-1}(x), \widetilde{v}_{n-1}(p x)\right), \\
\tilde{u}_{n}(x)=P_{n} M_{n}(x), \\
\mathscr{L} N_{n}(x)=G\left(x, \widetilde{v}_{n-1}(x), \tilde{u}_{n-1}(q x)\right), \\
\tilde{v}_{n}(x)=P_{n} N_{n}(x),
\end{gathered}
$$

where $M_{n}(x), N_{n}(x) \in W_{2}[0,+\infty)$ and $P_{n}: W_{2} \rightarrow$ $\operatorname{Span}\left\{\bar{\psi}_{1}, \bar{\psi}_{2}, \ldots, \bar{\psi}_{n}\right\}$ is a orthogonal projection operator. Then by (13) it followed that:

$$
\begin{aligned}
& M_{n}(x)=\int_{0}^{x} F\left(\mu, \widetilde{u}_{n-1}(\mu), \widetilde{v}_{n-1}(p \mu)\right) d \mu, \\
& M_{n}^{\prime}(x)=F\left(x, \widetilde{u}_{n-1}(x), \widetilde{v}_{n-1}(p x)\right), \\
& M_{n}^{\prime \prime}(x)=\frac{d}{d x} F\left(x, \tilde{u}_{n-1}(x), \widetilde{v}_{n-1}(p x)\right), \\
& N_{n}(x)=\int_{0}^{x} G\left(\mu, \widetilde{v}_{n-1}(\mu), \widetilde{u}_{n-1}(q \mu)\right) d \mu, \\
& N_{n}^{\prime}(x)=G\left(x, \widetilde{v}_{n-1}(x), \widetilde{u}_{n-1}(q x)\right), \\
& N_{n}^{\prime \prime}(x)=\frac{d}{d x} G\left(x, \widetilde{v}_{n-1}(x), \widetilde{u}_{n-1}(q x)\right) .
\end{aligned}
$$

Lemma 6. Let $\left\{x_{i}\right\}_{i=1}^{\infty}$ be dense on $[0,+\infty)$; if the solution of (2) is unique, then the solution satisfies the form

$$
\begin{gathered}
M_{n}(x)=\sum_{i=1}^{\infty} \sum_{k=1}^{i} \beta_{i k} F\left(x_{k}, \tilde{u}_{n-1}\left(x_{k}\right), \widetilde{v}_{n-1}\left(p x_{k}\right)\right) \bar{\psi}_{i}(x), \\
N_{n}(x)=\sum_{i=1}^{\infty} \sum_{k=1}^{i} \beta_{i k} G\left(x_{k}, \widetilde{v}_{n-1}\left(x_{k}\right), \tilde{u}_{n-1}\left(q x_{k}\right)\right) \bar{\psi}_{i}(x) .
\end{gathered}
$$

Proof. Note that $\left\langle u(x), \varphi_{i}(x)\right\rangle=u\left(x_{i}\right)$ and $\left\{\bar{\psi}_{i}(x)\right\}_{i=1}^{\infty}$ is an orthonormal basis of $W_{2}[0,+\infty)$; hence according to Lemma 5 we have

$$
\begin{aligned}
M_{n}(x) & =\sum_{i=1}^{\infty}\left\langle M_{n}(x), \bar{\psi}_{i}(x)\right\rangle \bar{\psi}_{i}(x) \\
& =\sum_{i=1}^{\infty}\left\langle M_{n}(x), \sum_{k=1}^{i} \beta_{i k} \psi_{k}(x)\right\rangle \bar{\psi}_{i}(x) \\
& =\sum_{i=1}^{\infty} \sum_{k=1}^{i} \beta_{i k}\left\langle M_{n}(x), \psi_{k}(x)\right\rangle \bar{\psi}_{i}(x) \\
& =\sum_{i=1}^{\infty} \sum_{k=1}^{i} \beta_{i k}\left\langle\mathscr{L} M_{n}(x), \varphi_{k}(x)\right\rangle \bar{\psi}_{i}(x) \\
& =\sum_{i=1}^{\infty} \sum_{k=1}^{i} \beta_{i k} F\left(x_{k}, u_{n-1}\left(x_{k}\right), v_{n-1}\left(p x_{k}\right)\right) \bar{\psi}_{i}(x) .
\end{aligned}
$$

In the same manner

$$
N_{n}(x)=\sum_{i=1}^{\infty} \sum_{k=1}^{i} \beta_{i k} G\left(x_{k}, v_{n-1}\left(x_{k}\right), u_{n-1}\left(q x_{k}\right)\right) \bar{\psi}_{i}(x) \text {. }
$$

Take $u_{0}(x)=v_{0}(x)=0$; define the iterative sequence

$$
\begin{aligned}
u_{n}(x) & =P_{n} M_{n}(x) \\
& =\sum_{i=1}^{n} \sum_{k=1}^{i} \beta_{i k} F\left(x_{k}, u_{n-1}\left(x_{k}\right), v_{n-1}\left(p x_{k}\right)\right) \bar{\psi}_{i}(x), \\
v_{n}(x) & =P_{n} N_{n}(x) \\
& =\sum_{i=1}^{n} \sum_{k=1}^{i} \beta_{i k} G\left(x_{k}, v_{n-1}\left(x_{k}\right), u_{n-1}\left(q x_{k}\right)\right) \bar{\psi}_{i}(x) .
\end{aligned}
$$

3.2. The Boundedness of Sequence $u_{n}(x)$ and $v_{n}(x)$. From

$$
\partial_{y}^{3} R_{x}(x+0)-\partial_{y}^{3} R_{x}(x-0)=1,
$$

we have

$$
\begin{aligned}
\partial_{y}^{4} R_{x}(y)= & g_{x}(y)+\left(\partial_{y}^{3} R_{x}(x+0)-\partial_{y}^{3} R_{x}(x-0)\right) \\
& \times \delta(x-y) \\
= & g_{x}(y)+\delta(x-y),
\end{aligned}
$$

where

$$
g_{x}(y)= \begin{cases}g_{x}^{1}, & y<x, \\ g_{x}^{2}, & y>x, \\ 0, & y=x\end{cases}
$$

$g_{x}^{1}(y)=\partial_{y}^{4}\left(a_{1} e^{y}+a_{2} e^{-y}+a_{3} e^{2 y}+a_{4} e^{-2 y}\right) ; g_{x}^{2}(y)=\partial_{y}^{4}\left(b_{1} e^{y}+\right.$ $\left.b_{2} e^{-y}+b_{3} e^{2 y}+b_{4} e^{-2 y}\right)$; and $g_{x}^{1}(y), g_{x}^{2}(y), \partial_{y} g_{x}^{1}(y), \partial_{y} g_{x}^{2}(y)$ are bounded functions with respect to $x, y$, respectively. 
Lemma 7. For $x \in[0,+\infty), y, z \in(-\infty,+\infty)$, and $F(x, y, z)$, $G(x, y, z)$ are continuous bounded functions on $[0,+\infty)$, we have that $M_{n}^{(i)}(x)$ and $N_{n}^{(i)}(x)(i=0,1,2)$ are bounded.

Proof. By the expression of $M_{n}(x), M_{n}^{\prime}(x)$ and the assumptions, we know that $M_{n}(x)$ and $M_{n}^{\prime}(x)$ are bounded. In the following, we will discuss the boundedness of $M_{n}^{\prime \prime}(x)$.

Since the function which is in $C_{[0,+\infty)}^{2}$ is dense in $W_{2}[0,+\infty)$, without loss of generality, we assume that $M_{n}^{\prime \prime}(x)$ is continuous.

Note that

$$
4 R_{x}(y)-5 \partial_{y}^{2} R_{x}(y)+\partial_{y}^{4} R_{x}(y)=\delta(x-y) .
$$

One gets

$$
\begin{aligned}
M_{n}^{\prime \prime}(x)= & \int_{0}^{\infty} M_{n}^{\prime \prime}(y) \delta(x-y) d y \\
= & \int_{0}^{\infty} M_{n}^{\prime \prime}(y)\left[4 R_{x}(y)-5 \partial_{y}^{2} R_{x}(y)+\partial_{y}^{4} R_{x}(y)\right] d y \\
= & \int_{0}^{\infty} M_{n}^{\prime \prime}(y)\left[4 R_{x}(y)-5 \partial_{y}^{2} R_{x}(y)\right] d y \\
& +\int_{0}^{\infty} M_{n}^{\prime \prime}(y) \partial_{y}^{4} R_{x}(y) d y \\
= & \left.M_{n}^{\prime}(y)\left[4 R_{x}(y)-5 \partial_{y}^{2} R_{x}(y)\right]\right|_{0} ^{\infty} \\
& -\int_{0}^{+\infty} M_{n}^{\prime}(y)\left[4 \partial_{y} R_{x}(y)-5 \partial_{y}^{3} R_{x}(y)\right] d y \\
& +\int_{0}^{\infty} M_{n}^{\prime \prime}(y)\left[g_{x}(y)+\delta(x-y)\right] d y \\
= & m(x)+\int_{0}^{\infty} M_{n}^{\prime \prime}(y)\left[g_{x}(y)+\delta(x-y)\right] d y \\
= & m(x)+\int_{0}^{\infty} M_{n}^{\prime \prime}(y) g_{x}(y) d y+M_{n}^{\prime \prime}(x) \\
& \\
& \\
&
\end{aligned}
$$

where $m(x)=\left.M_{n}^{\prime}(y)\left[4 R_{x}(y)-5 \partial_{y}^{2} R_{x}(y)\right]\right|_{0} ^{\infty}-$ $\int_{0}^{\infty} M_{n}^{\prime}(y)\left[4 \partial_{y} R_{x}(y)-5 \partial_{y}^{3} R_{x}(y)\right] d y$. Thus, we have

$$
\begin{aligned}
0= & m(x)-\int_{0}^{\infty} M_{n}^{\prime \prime}(y) g_{x}(y) d y \\
= & m(x)-\int_{0}^{x} M_{n}^{\prime \prime}(y) g_{x}^{1}(y) d y-\int_{x}^{\infty} M_{n}^{\prime \prime}(y) g_{x}^{2}(y) d y \\
= & m(x)-\left.M_{n}^{\prime}(y) g_{x}^{1}(y)\right|_{0} ^{x}+\int_{0}^{x} M_{n}^{\prime}(y) \partial_{y} g_{x}^{1}(y) d y \\
& -\left.M_{n}^{\prime}(y) g_{x}^{2}(y)\right|_{x} ^{M}+\int_{x}^{\infty} M_{n}^{\prime}(y) \partial_{y} g_{x}^{2}(y) d y
\end{aligned}
$$

$$
\begin{aligned}
=m & (x)-M_{n}^{\prime}(x)\left(g_{x}^{1}(x)-g_{x}^{2}(x)\right) \\
& +\left(M_{n}^{\prime}(0) g_{x}^{1}(0)-M_{n}^{\prime}(M) g_{x}^{2}(M)\right) \\
& +\int_{0}^{x} M_{n}^{\prime}(y) \partial_{y} g_{x}^{1}(y) d y \\
& +\int_{x}^{\infty} M_{n}^{\prime}(y) \partial_{y} g_{x}^{2}(y) d y .
\end{aligned}
$$

So

$$
\begin{aligned}
M_{n}^{\prime}(x) & \left(g_{x}^{1}(x)-g_{x}^{2}(x)\right) \\
= & m(x)+\left(M_{n}^{\prime}(0) g_{x}^{1}(0)-M_{n}^{\prime}(M) g_{x}^{2}(M)\right) \\
& +\int_{0}^{x} M_{n}^{\prime}(y) \partial_{y} g_{x}^{1}(y) \\
& +\int_{x}^{\infty} M_{n}^{\prime}(y) \partial_{y} g_{x}^{2}(y) d y .
\end{aligned}
$$

Furthermore, we see that

$$
\begin{aligned}
M_{n}^{\prime \prime}(x)= & \frac{1}{g_{x}^{1}(x)-g_{x}^{2}(x)} \\
& \times\left\{\partial _ { x } \left[m(x)+\left(M_{n}^{\prime}(0) g_{x}^{1}(0)-M_{n}^{\prime}(M) g_{x}^{2}(M)\right)\right.\right. \\
& +\int_{0}^{x} M_{n}^{\prime}(y) \partial_{y} g_{x}^{1}(y) \\
& \left.+\int_{x}^{\infty} M_{n}^{\prime}(y) \partial_{y} g_{x}^{2}(y) d y\right] \\
& \left.-M_{n}^{\prime}(x) \partial_{x}\left(g_{x}^{1}(x)-g_{x}^{2}(x)\right)\right\} .
\end{aligned}
$$

In view of the expression of $R_{x}(y)$, we know that $\partial_{y}^{m} R_{x}(y)$ are bounded for $m=1,2,3$. It follows that $M_{n}^{\prime \prime}(x)$ is bounded from the boundedness of $g_{x}^{1}(y), g_{x}^{2}(y), \partial_{y} g_{x}^{1}(y), \partial_{y} g_{x}^{2}(y)$, and $M_{n}^{\prime}(x)$. In the same way, $N_{n}(x), N_{n}^{\prime}(x)$, and $N_{n}^{\prime \prime}(x)$ are bounded.

Lemma 8. Assume that $F(x, y, z), G(x, y, z)$ are continuous bounded functions for $x \in[0,+\infty), y, z \in(-\infty,+\infty)$, and $F(x, y, z), G(x, y, z) \in W_{1}[0,+\infty)$ as $y=y(x) \in W_{2}, z=$ $z(x) \in W_{2}$, then

$$
\left\|u_{n}\right\|_{W_{2}} \leq C, \quad\left\|v_{n}\right\|_{W_{2}} \leq D, \quad(C, D \text { are constants })
$$


Proof.

$$
\left\|M_{n}\right\|_{W_{2}}^{2}=\left\langle M_{n}, M_{n}\right\rangle=\int_{0}^{\infty}\left(4 M_{n}^{2}+5 M_{n}^{\prime 2}+M_{n}^{\prime \prime 2}\right) d x .
$$

Note that (14) and the assumptions, by Lemma 7, $\left|M_{n}^{(i)}(x)\right| \leq$ $C^{i}, i=0,1,2$, thus $\left\|M_{n}\right\| \leq C$, where $C=\max \left\{C^{0}, C^{1}, C^{2}\right\}$, from (19), we have $\left\|u_{n}\right\|_{W_{2}} \leq\left\|M_{n}\right\|_{W_{2}} \leq C$. In the same way, we obtain $\left\|v_{n}\right\|_{W_{2}} \leq D$.

\subsection{Construction the of Another Iterative Sequence}

$$
u_{n}(x) \text { and } v_{n}(x)
$$

Theorem 9. Let $\left\{x_{i}\right\}_{i=1}^{\infty}$ be dense on $[0,+\infty)$; if the solution of (2) exists and unique, then the solution satisfies the form

$$
\begin{aligned}
& u(x)=\sum_{i=1}^{\infty} \sum_{k=1}^{i} \beta_{i k} F\left(x_{k}, u\left(x_{k}\right), v\left(p x_{k}\right)\right) \bar{\psi}_{i}(x), \\
& v(x)=\sum_{i=1}^{\infty} \sum_{k=1}^{i} \beta_{i k} G\left(x_{k}, v\left(x_{k}\right), u\left(q x_{k}\right)\right) \bar{\psi}_{i}(x) .
\end{aligned}
$$

Proof. Note that $\left\langle u(x), \varphi_{i}(x)\right\rangle=u\left(x_{i}\right)$ and $\left\{\bar{\psi}_{i}(x)\right\}_{i=1}^{\infty}$ is an orthonormal basis of $W_{2}[0,+\infty)$; hence we have

$$
\begin{aligned}
u(x) & =\sum_{i=1}^{\infty}\left\langle u(x), \bar{\psi}_{i}(x)\right\rangle \bar{\psi}_{i}(x) \\
& =\sum_{i=1}^{\infty}\left\langle u(x), \sum_{k=1}^{i} \beta_{i k} \psi_{k}(x)\right\rangle \bar{\psi}_{i}(x) \\
& =\sum_{i=1}^{\infty} \sum_{k=1}^{i} \beta_{\mathrm{i} k}\left\langle u(x), \psi_{k}(x)\right\rangle \bar{\psi}_{i}(x) \\
& =\sum_{i=1}^{\infty} \sum_{k=1}^{i} \beta_{i k}\left\langle\mathscr{L} u(x), \varphi_{k}(x)\right\rangle \bar{\psi}_{i}(x) \\
& =\sum_{i=1}^{\infty} \sum_{k=1}^{i} \beta_{i k} F\left(x_{k}, u\left(x_{k}\right), v\left(p x_{k}\right)\right) \bar{\psi}_{i}(x) .
\end{aligned}
$$

In the same manner

$$
v(x)=\sum_{i=1}^{\infty} \sum_{k=1}^{i} \beta_{i k} G\left(x_{k}, v\left(x_{k}\right), u\left(q x_{k}\right)\right) \bar{\psi}_{i}(x) .
$$

In the following, a new method of solving (2) is presented. Equations (31) and (32) can be denoted by

$$
\begin{aligned}
& u(x)=\sum_{i=1}^{\infty} A_{i} \overline{\psi_{i}}(x), \\
& v(x)=\sum_{i=1}^{\infty} B_{i} \overline{\psi_{i}}(x),
\end{aligned}
$$

where $A_{i}=\sum_{k=1}^{i} \beta_{i k} F\left(x_{k}, u\left(x_{k}\right), v\left(p x_{k}\right)\right)$ and $B_{i}=$ $\sum_{k=1}^{i} \beta_{i k} G\left(x_{k}, v\left(x_{k}\right), u\left(q x_{k}\right)\right)$. In fact, $A_{i}$ and $B_{i}$ are unknown; we will approximate $A_{i}$ and $B_{i}$ by using the known $\bar{A}_{i}$ and $\bar{B}_{i}$. We take $u_{1}(x)=0, v_{1}(x)=0$ and define the following iterative sequence

$$
\begin{aligned}
& u_{n}(x)=\sum_{i=1}^{n-1} \bar{A}_{i} \overline{\psi_{i}}(x), \\
& v_{n}(x)=\sum_{i=1}^{n-1} \bar{B}_{i} \overline{\psi_{i}}(x),
\end{aligned}
$$

where

$$
\begin{aligned}
& \bar{A}_{1}=\beta_{11} F\left(x_{1}, u_{1}\left(x_{1}\right), v_{1}\left(p x_{1}\right)\right) \\
& \bar{A}_{2}=\sum_{k=1}^{2} \beta_{2 k} F\left(x_{k}, u_{k}\left(x_{k}\right), v_{k}\left(p x_{k}\right)\right) \\
& \vdots
\end{aligned}
$$

$$
\begin{gathered}
\bar{A}_{n-1}=\sum_{k=1}^{n-1} \beta_{n k} F\left(x_{k}, u_{k}\left(x_{k}\right), v_{k}\left(p x_{k}\right)\right), \\
\bar{B}_{1}=\beta_{11} G\left(x_{1}, v_{1}\left(x_{1}\right), u_{1}\left(q x_{1}\right)\right) \\
\bar{B}_{2}=\sum_{k=1}^{2} \beta_{2 k} G\left(x_{k}, v_{k}\left(x_{k}\right), u_{k}\left(q x_{k}\right)\right) \\
\vdots \\
\bar{B}_{n-1}=\sum_{k=1}^{n-1} \beta_{n k} G\left(x_{k}, v_{k}\left(x_{k}\right), u_{k}\left(q x_{k}\right)\right) .
\end{gathered}
$$

Next, lemmas are given.

Lemma 10. The following iterative sequences

$$
\begin{aligned}
& u_{n}(x)=\sum_{i=1}^{n-1} \beta_{i k} F\left(x_{k}, u_{k}\left(x_{k}\right), v_{k}\left(p x_{k}\right)\right) \overline{\psi_{i}}(x), \\
& v_{n}(x)=\sum_{i=1}^{n-1} \beta_{i k} G\left(x_{k}, v_{k}\left(x_{k}\right), u_{k}\left(q x_{k}\right)\right) \overline{\psi_{i}}(x)
\end{aligned}
$$

satisfy

$$
\begin{aligned}
& \mathscr{L} u_{n}\left(x_{j}\right)=F\left(x_{j}, u_{j}\left(x_{j}\right), v_{j}\left(p x_{j}\right)\right), \\
& \mathscr{L} v_{n}\left(x_{j}\right)=G\left(x_{j}, v_{j}\left(x_{j}\right), u_{j}\left(q x_{j}\right)\right), \quad j \leq n-1,
\end{aligned}
$$

respectively. 
Proof. If $j=1$,

$$
\begin{aligned}
\mathscr{L} u_{n}\left(x_{1}\right)= & \sum_{i=1}^{n-1} \beta_{i k} F\left(x_{k}, u_{k}\left(x_{k}\right), v_{k}\left(p x_{k}\right)\right) \mathscr{L} \bar{\psi}_{i}\left(x_{1}\right) \\
= & \sum_{i=1}^{n-1} \beta_{i k} F\left(x_{k}, u_{k}\left(x_{k}\right), v_{k}\left(p x_{k}\right)\right) \\
& \times\left\langle\mathscr{L} \bar{\psi}_{i}(x), \varphi_{1}(x)\right\rangle \\
= & \sum_{i=1}^{n-1} \beta_{i k} F\left(x_{k}, u_{k}\left(x_{k}\right), v_{k}\left(p x_{k}\right)\right) \\
& \times\left\langle\bar{\psi}_{i}(x), \psi_{1}(x)\right\rangle, \\
\beta_{11} \mathscr{L} u_{n}\left(x_{1}\right)= & \sum_{i=1}^{n-1} \beta_{i k} F\left(x_{k}, u_{k}\left(x_{k}\right), v_{k}\left(p x_{k}\right)\right) \\
& \times\left\langle\bar{\psi}_{i}(x), \bar{\psi}_{1}(x)\right\rangle \\
= & \beta_{11} F\left(x_{1}, u_{1}\left(x_{1}\right), v_{1}\left(p x_{1}\right)\right),
\end{aligned}
$$

so,

$$
\mathscr{L} u_{n}\left(x_{1}\right)=F\left(x_{1}, u_{1}\left(x_{1}\right), v_{1}\left(p x_{1}\right)\right) .
$$

If $j=2$,

$$
\begin{aligned}
\mathscr{L} u_{n}\left(x_{2}\right) & =\sum_{i=1}^{n-1} \beta_{i k} F\left(x_{k}, u_{k}\left(x_{k}\right), v_{k}\left(p x_{k}\right)\right) \mathscr{L} \bar{\psi}_{i}\left(x_{2}\right) \\
& =\sum_{i=1}^{n-1} \beta_{i k} F\left(x_{k}, u_{k}\left(x_{k}\right), v_{k}\left(p x_{k}\right)\right)\left\langle\bar{\psi}_{i}(x), \psi_{2}(x)\right\rangle,
\end{aligned}
$$

$\beta_{21} \times(40)+\beta_{22} \times(42)$, we have

$$
\begin{aligned}
\beta_{21} \mathscr{L} u_{n} & \left(x_{1}\right)+\beta_{22} \mathscr{L} u_{n}\left(x_{2}\right) \\
= & \sum_{i=1}^{n-1} \beta_{i k} F\left(x_{k}, u_{k}\left(x_{k}\right), v_{k}\left(p x_{k}\right)\right)\left\langle\bar{\psi}_{i}(x), \bar{\psi}_{2}(x)\right\rangle \\
= & \beta_{21} F\left(x_{1}, u_{1}\left(x_{1}\right), v_{1}\left(p x_{1}\right)\right) \\
& +\beta_{22} F\left(x_{2}, u_{2}\left(x_{2}\right), v_{2}\left(p x_{2}\right)\right),
\end{aligned}
$$

by (41), $\mathscr{L} u_{n}\left(x_{2}\right)=F\left(x_{2}, u_{2}\left(x_{2}\right), v_{2}\left(p x_{2}\right)\right)$. In the same way, we have

$$
\mathscr{L} u_{n}\left(x_{j}\right)=F\left(x_{j}, u_{j}\left(x_{j}\right), v_{j}\left(p x_{j}\right)\right), \quad j \leq n-1 .
$$

Similarly,

$$
\mathscr{L} v_{n}\left(x_{j}\right)=F\left(x_{j}, v_{j}\left(x_{j}\right), u_{j}\left(q x_{j}\right)\right), \quad j \leq n-1 .
$$

Theorem 11. The iterative form

$$
\begin{aligned}
& \widetilde{u}_{n}(x)=\sum_{i=1}^{n} \sum_{k=1}^{i} \beta_{i k} F\left(x_{k}, \widetilde{u}_{n-1}\left(x_{k}\right), \widetilde{v}_{n-1}\left(p x_{k}\right)\right) \bar{\psi}_{i}(x), \\
& \widetilde{v}_{n}(x)=\sum_{i=1}^{n} \sum_{k=1}^{i} \beta_{i k} G\left(x_{k}, \widetilde{v}_{n-1}\left(x_{k}\right), \widetilde{u}_{n-1}\left(q x_{k}\right)\right) \bar{\psi}_{i}(x), \\
& \widetilde{u}_{1}(0)=\widetilde{v}_{1}(0)=0
\end{aligned}
$$

and the iterative form

$$
\begin{aligned}
& u_{n}(x)=\sum_{i=1}^{n-1} \sum_{k=1}^{i} \beta_{i k} F\left(x_{k}, u_{k}\left(x_{k}\right), v_{k}\left(p x_{k}\right)\right) \bar{\psi}_{i}(x), \\
& v_{n}(x)=\sum_{i=1}^{n-1} \sum_{k=1}^{i} \beta_{i k} G\left(x_{k}, v_{k}\left(x_{k}\right), u_{k}\left(q x_{k}\right)\right) \bar{\psi}_{i}(x), \\
& u_{1}(0)=v_{1}(0)=0
\end{aligned}
$$

are the same.

Proof. In Lemma 10, let $n-1=k$; then

$$
\mathscr{L} u_{k+1}\left(x_{k}\right)=F\left(x_{k}, u_{k}\left(x_{k}\right), v_{k}\left(p x_{k}\right)\right),
$$

but

$$
\mathscr{L} u_{n}\left(x_{k}\right)=F\left(x_{k}, u_{k}\left(x_{k}\right), v_{k}\left(p x_{k}\right)\right),
$$

thus

$$
\begin{aligned}
u_{n}(x) & =\sum_{i=1}^{n-1} \sum_{k=1}^{i} \beta_{i k} L u_{k+1}\left(x_{k}\right) \bar{\psi}_{i}(x) \\
& =\sum_{i=1}^{n-1} \sum_{k=1}^{i} \beta_{i k} \mathscr{L} u_{n}\left(x_{k}\right) \bar{\psi}_{i}(x) \\
& =\sum_{i=1}^{n-1} \sum_{k=1}^{i} \beta_{i k}\left\langle\mathscr{L} u_{n}(x), \varphi_{k}(x)\right\rangle \bar{\psi}_{i}(x) \\
& =\sum_{i=1}^{n-1} \sum_{k=1}^{i} \beta_{i k}\left\langle u_{n}(x), \psi_{k}(x)\right\rangle \bar{\psi}_{i}(x) \\
& =\sum_{i=1}^{n-1}\left\langle u_{n}(x), \bar{\psi}_{i}(x)\right\rangle \bar{\psi}_{i}(x) .
\end{aligned}
$$

Equation (46) can be written as

$$
\begin{gathered}
\mathscr{L} M_{n}(x)=F\left(x, \tilde{u}_{n-1}(x), \widetilde{v}_{n-1}(p x)\right), \\
\tilde{u}_{n}(x)=P_{n} M_{n}(x) .
\end{gathered}
$$


In fact, $M_{n}(x)=\sum_{i=1}^{\infty} \sum_{k=1}^{i} \beta_{i k} F\left(x_{k}, \widetilde{u}_{n-1}\left(x_{k}\right), \widetilde{v}_{n-1}\left(p x_{k}\right)\right) \bar{\psi}_{i}(x)$; then

$$
\begin{aligned}
\mathscr{L} \widetilde{u}_{n}\left(x_{j}\right) & =\mathscr{L} P_{n} M_{n}\left(x_{j}\right) \\
& =\left\langle\mathscr{L} P_{n} M_{n}(x), \varphi_{j}(x)\right\rangle \\
& =\left\langle P_{n} M_{n}(x), \psi_{j}(x)\right\rangle \\
& =\left\langle M_{n}(x), \psi_{j}(x)\right\rangle \\
& =\left\langle\mathscr{L} M_{n}(x), \varphi_{j}(x)\right\rangle \\
& =\mathscr{L} M_{n}\left(x_{j}\right),
\end{aligned}
$$

by (51) and (52), we have

$$
\begin{aligned}
\tilde{u}_{n}(x) & =\sum_{i=1}^{n-1} \sum_{k=1}^{i} \beta_{i k} F\left(x_{k}, \tilde{u}_{n-1}\left(x_{k}\right), \tilde{v}_{n-1}\left(p x_{k}\right)\right) \bar{\psi}_{i}(x) \\
& =\sum_{i=1}^{n-1} \sum_{k=1}^{i} \beta_{i k} \mathscr{L} M_{n}\left(x_{k}\right) \bar{\psi}_{i}(x) \\
& =\sum_{i=1}^{n-1} \sum_{k=1}^{i} \beta_{i k} \mathscr{L} \widetilde{u}_{n}\left(x_{k}\right) \bar{\psi}_{i}(x) \\
& =\sum_{i=1}^{n-1} \sum_{k=1}^{i} \beta_{i k}\left\langle\mathscr{L} \tilde{u}_{n}(x), \varphi_{k}(x)\right\rangle \bar{\psi}_{i}(x) \\
& =\sum_{i=1}^{n-1} \sum_{k=1}^{i} \beta_{i k}\left\langle\widetilde{u}_{n}(x), \psi_{k}(x)\right\rangle \bar{\psi}_{i}(x) \\
& =\sum_{i=1}^{n-1}\left\langle\widetilde{u}_{n}(x), \bar{\psi}_{i}(x)\right\rangle \bar{\psi}_{i}(x) .
\end{aligned}
$$

Equation (53) is the same as (50). We may prove for $\widetilde{v}_{n}(x)$ similarly.

So, by Theorem 11 and Lemma 8, we have the following Theorem.

Theorem 12. Under the conditions of Lemma 8,

$$
\begin{aligned}
& u_{n}(x)=\sum_{i=1}^{n-1} \beta_{i k} F\left(x_{k}, u_{k}\left(x_{k}\right), v_{k}\left(p x_{k}\right)\right) \overline{\psi_{i}}(x), \\
& v_{n}(x)=\sum_{i=1}^{n-1} \beta_{i k} G\left(x_{k}, v_{k}\left(x_{k}\right), u_{k}\left(q x_{k}\right)\right) \overline{\psi_{i}}(x)
\end{aligned}
$$

satisfy $\left\|u_{n}(x)\right\| \leq C,\left\|v_{n}(x)\right\| \leq D$.

Lemma 13. If $u(x)$ and $v(x) \in W_{2}[0,+\infty)$, then there exists $M_{1}, M_{2}>0$, such that $|u(x)| \leq M_{1}\|u(x)\|_{W_{2}}$ and $|v(x)| \leq$ $M_{2}\|v(x)\|_{W_{2}}$.

Proof. It is easy to obtain from the properties in the reproducing kernel space.
By Lemma 13 and Theorem 12, it is easy to obtain the following Lemma 14.

Lemma 14. If $u_{n}(x) \stackrel{\|\cdot\|_{W_{2}}}{\longrightarrow} \bar{u}(x)(n \rightarrow \infty), v_{n}(x) \stackrel{\|\cdot\|_{W_{2}}}{\longrightarrow}$ $\bar{v}(x)(n \rightarrow \infty), x_{n} \rightarrow y(n \rightarrow \infty)$, and $F(x, y, z)$ and $G(x, y, z)$ satisfy the conditions of Lemma 8 , then

$$
\begin{aligned}
& F\left(x_{n}, u_{n-1}\left(x_{n}\right), v_{n-1}\left(p x_{n}\right)\right) \\
& \quad \longrightarrow F(y, \bar{u}(y), \bar{v}(p y)) \text { as } n \longrightarrow \infty, \\
& G\left(x_{n}, v_{n-1}\left(x_{n}\right), u_{n-1}\left(q x_{n}\right)\right) \\
& \quad \longrightarrow G(y, \bar{v}(y), \bar{u}(q y)) \text { as } n \longrightarrow \infty .
\end{aligned}
$$

Theorem 15. Let $\left\{x_{i}\right\}_{i=1}^{\infty}$ be dense in $[0,+\infty)$, and $F(x, y, z)$ and $G(x, y, z)$ satisfy the conditions of Lemma 8 , then the $n$ term approximate solutions $u_{n}(x)$ and $v_{n}(x)$ in (35) converge to the exact solution $u(x)$ and $v(x)$ of $(2)$, respectively, and $u(x)=$ $\sum_{i=1}^{\infty} \bar{A}_{i} \bar{\psi}_{i}(x), v(x)=\sum_{i=1}^{\infty} \bar{B}_{i} \bar{\psi}_{i}(x)$, where $\bar{A}_{i}$ and $\bar{B}_{i}$ are given, respectively, by (36), and (37).

Proof. (1) Firstly, we will prove the convergence of $u_{n}(x), v_{n}(x)$.

By (35), we infer that

$$
\begin{aligned}
& u_{n+1}(x)=u_{n}(x)+\bar{A}_{n} \bar{\psi}_{n}(x), \\
& v_{n+1}(x)=v_{n}(x)+\bar{B}_{n} \bar{\psi}_{n}(x) .
\end{aligned}
$$

From the orthogonality of $\left\{\bar{\psi}_{i}(x)\right\}_{i=1}^{\infty}$, it follows that

$$
\begin{aligned}
\left\|u_{n+1}\right\|_{W_{2}}^{2} & =\left\|u_{n}\right\|_{W_{2}}^{2}+\left(\bar{A}_{n}\right)^{2} \\
& =\left\|u_{n-1}\right\|_{W_{2}}^{2}+\left(\bar{A}_{n-1}\right)^{2}+\left(\bar{A}_{n}\right)^{2} \\
& \vdots \\
& =\left\|u_{1}\right\|_{W_{2}}^{2}+\sum_{i=1}^{n}\left(\bar{A}_{i}\right)^{2}, \\
\left\|v_{n+1}\right\|_{W_{2}}^{2} & =\left\|v_{n}\right\|_{W_{2}}^{2}+\left(\bar{B}_{n}\right)^{2} \\
& =\left\|v_{n-1}\right\|_{W_{2}}^{2}+\left(\bar{B}_{n-1}\right)^{2}+\left(\bar{B}_{n}\right)^{2} \\
& \vdots \\
& =\left\|v_{1}\right\|_{W_{2}}^{2}+\sum_{i=1}^{n}\left(\bar{B}_{i}\right)^{2} .
\end{aligned}
$$

From (59) and (60), we know sequence $\left\|u_{n}\right\|_{W_{2}}$ and $\left\|v_{n}\right\|_{W_{2}}$ is increasing. By Theorem 12, $\left\|u_{n}\right\|_{W_{2}}$ and $\left\|v_{n}\right\|_{W_{2}}$ are bounded; hence $\left\|u_{n}\right\|_{W_{2}}$ and $\left\|v_{n}\right\|_{W_{2}}$ are convergent such that

$$
\begin{aligned}
& \sum_{i=1}^{\infty}\left(\bar{A}_{i}\right)^{2}<\infty, \\
& \sum_{i=1}^{\infty}\left(\bar{B}_{i}\right)^{2}<\infty .
\end{aligned}
$$


This implies that

$$
\begin{aligned}
& \bar{A}_{i}=\sum_{k=1}^{i} \beta_{i k} F\left(x_{k}, u_{k}\left(x_{k}\right), v_{k}\left(p x_{k}\right)\right) \in l^{2} \quad(i=1,2, \ldots), \\
& \bar{B}_{i}=\sum_{k=1}^{i} \beta_{i k} G\left(x_{k}, v_{k}\left(x_{k}\right), u_{k}\left(q x_{k}\right)\right) \in l^{2} \quad(i=1,2, \ldots) .
\end{aligned}
$$

Without loss of generality, assume $m>n$; we have

$$
\begin{aligned}
\left\|u_{m}(x)-u_{n}(x)\right\|_{W_{2}}^{2}=\| & u_{m}(x)-u_{m-1}(x) \\
& +u_{m-1}(x)-u_{m-2}(x) \\
& +\cdots+u_{n+1}(x)-u_{n}(x) \|_{W_{2}}^{2} \\
\leq & \left\|u_{m}(x)-u_{m-1}(x)\right\|_{W_{2}}^{2} \\
& +\cdots+\left\|u_{n+1}(x)-u_{n}(x)\right\|_{W_{2}}^{2} \\
= & \sum_{i=n+1}^{m}\left(\bar{A}_{i}\right)^{2} \longrightarrow 0, \quad(n \longrightarrow \infty), \\
\left\|v_{m}(x)-v_{n}(x)\right\|_{W_{2}}^{2}= & \| v_{m}(x)-v_{m-1}(x) \\
& +v_{m-1}(x)-v_{m-2}(x) \\
& +\cdots+v_{n+1}(x)-v_{n}(x) \|_{W_{2}}^{2} \\
\leq & \left\|v_{m}(x)-v_{m-1}(x)\right\|_{W_{2}}^{2} \\
& +\ldots+\left\|v_{n+1}(x)-v_{n}(x)\right\|_{W_{2}}^{2} \\
= & \left.\sum_{i=n+1}^{m}\left(\bar{B}_{i}\right)^{2} \longrightarrow \infty\right) .
\end{aligned}
$$

Considering the completeness of $W_{2}[0,+\infty)$, there exist $\bar{u}(x)$ and $\bar{v}(x)$ in $W_{2}[0,+\infty)$ such that

$$
\begin{aligned}
& u_{n}(x) \stackrel{\|\cdot\|}{\longrightarrow} \bar{u}(x) \quad \text { as } n \longrightarrow \infty, \\
& v_{n}(x) \stackrel{\|\cdot\|}{\longrightarrow} \bar{v}(x) \text { as } n \longrightarrow \infty .
\end{aligned}
$$

(2) Secondly, we will prove that $\bar{u}(x)$ and $\bar{v}(x)$ are the solutions of (2).

By Lemma 14 and the proof of (1), we may know that $u_{n}(x)$, and $v_{n}(x)$ respectively, converge uniformly to $\bar{u}(x)$ and $\bar{v}(x) \quad(n \rightarrow \infty)$. Taking limits in (35), we have

$$
\begin{aligned}
& \bar{u}(x)=\sum_{i=1}^{\infty} \bar{A}_{i} \bar{\psi}_{i}(x), \\
& \bar{v}(x)=\sum_{i=1}^{\infty} \bar{B}_{i} \bar{\psi}_{i}(x) .
\end{aligned}
$$

Since

$$
\begin{aligned}
(\mathscr{L} \bar{u})\left(x_{j}\right) & =\sum_{i=1}^{\infty} \bar{A}_{i}\left\langle\mathscr{L} \bar{\psi}_{i}, \varphi_{j}\right\rangle \\
& =\sum_{i=1}^{\infty} \bar{A}_{i}\left\langle\bar{\psi}_{i}, \mathscr{L}^{*} \varphi_{j}\right\rangle \\
& =\sum_{i=1}^{\infty} \bar{A}_{i}\left\langle\bar{\psi}_{i}, \psi_{j}\right\rangle,
\end{aligned}
$$

in the same way, we have

$$
(\mathscr{L} \bar{v})\left(x_{j}\right)=\sum_{i=1}^{\infty} \bar{B}_{i}\left\langle\bar{\psi}_{i}, \psi_{j}\right\rangle,
$$

it follows that

$$
\begin{aligned}
\sum_{j=1}^{n} \beta_{n j}(\mathscr{L} \bar{u})\left(x_{j}\right) & =\sum_{i=1}^{\infty} \bar{A}_{i}\left\langle\bar{\psi}_{i}, \sum_{j=1}^{n} \beta_{n j} \psi_{j}\right\rangle \\
& =\sum_{i=1}^{\infty} \bar{A}_{i}\left\langle\bar{\psi}_{i}, \bar{\psi}_{n}\right\rangle \\
& =\bar{A}_{n}, \\
\sum_{j=1}^{n} \beta_{n j}(\mathscr{L} \bar{v})\left(x_{j}\right) & =\sum_{i=1}^{\infty} \bar{B}_{i}\left\langle\bar{\psi}_{i}, \sum_{j=1}^{n} \beta_{n j} \psi_{j}\right\rangle \\
& =\sum_{i=1}^{\infty} \bar{B}_{i}\left\langle\bar{\psi}_{i}, \bar{\psi}_{n}\right\rangle \\
& =\bar{B}_{n} .
\end{aligned}
$$

If $n=1$, then

$$
\begin{aligned}
& (\mathscr{L} \bar{u})\left(x_{1}\right)=F\left(x_{1}, u_{1}\left(x_{1}\right), v_{1}\left(p x_{1}\right)\right) \\
& (\mathscr{L} \bar{v})\left(x_{1}\right)=G\left(x_{1}, v_{1}\left(x_{1}\right), u_{1}\left(q x_{1}\right)\right)
\end{aligned}
$$

If $n=2$, then

$$
\begin{aligned}
& \beta_{21}(\mathscr{L} \bar{u})\left(x_{1}\right)+\beta_{22}(\mathscr{L} \bar{u})\left(x_{2}\right) \\
&= \beta_{21} F\left(x_{1}, u_{1}\left(x_{1}\right), v_{1}\left(p x_{1}\right)\right) \\
&+\beta_{22} F\left(x_{2}, u_{2}\left(x_{2}\right), v_{2}\left(p x_{2}\right)\right), \\
& \beta_{21}(\mathscr{L} \bar{v})\left(x_{1}\right)+\beta_{22}(\mathscr{L} \bar{v})\left(x_{2}\right) \\
&=\beta_{21} G\left(x_{1}, v_{1}\left(x_{1}\right), u_{1}\left(q x_{1}\right)\right) \\
& \quad+\beta_{22} G\left(x_{2}, v_{2}\left(x_{2}\right), u_{2}\left(q x_{2}\right)\right) .
\end{aligned}
$$

From (70) and (71), it is clear that

$$
\begin{aligned}
& (\mathscr{L} \bar{u})\left(x_{2}\right)=F\left(x_{2}, u_{2}\left(x_{2}\right), v_{2}\left(p x_{2}\right)\right) \\
& (\mathscr{L} \bar{v})\left(x_{2}\right)=G\left(x_{2}, v_{2}\left(x_{2}\right), u_{2}\left(q x_{2}\right)\right) .
\end{aligned}
$$


Furthermore, it is easy to see by induction that

$$
\begin{aligned}
& (\mathscr{L} \bar{u})\left(x_{j}\right)=F\left(x_{j}, u_{j}\left(x_{j}\right), v_{j}\left(p x_{j}\right)\right), \quad j=1,2, \ldots, \\
& (\mathscr{L} \bar{v})\left(x_{j}\right)=G\left(x_{j}, v_{j}\left(x_{j}\right), u_{j}\left(q x_{j}\right)\right), \quad j=1,2, \ldots
\end{aligned}
$$

Since $\left\{x_{i}\right\}_{i=1}^{\infty}$ is dense in $[0,+\infty)$, for any $y \in[0,+\infty)$, there exists subsequence $\left\{x_{n_{j}}\right\}$ such that

$$
x_{n_{j}} \longrightarrow y \quad \text { as } j \longrightarrow \infty \text {. }
$$

Hence, let $j \rightarrow \infty$ in (74), and (75); by Lemma 14 and the continuity of $F(x, u(x), v(p x))$ and $G(x, v(x), u(q x))$, we have

$$
\begin{aligned}
& (\mathscr{L} \bar{u})(y)=F(y, \bar{u}(y), \bar{v}(p y)), \\
& (\mathscr{L} \bar{v})(y)=G(y, \bar{v}(y), \bar{u}(q y)) .
\end{aligned}
$$

That is, $\bar{u}(x)$ and $\bar{v}(x)$ are the solutions of (2) and

$$
\begin{aligned}
& \bar{u}(x)=\sum_{i=1}^{\infty} \bar{A}_{i} \bar{\psi}_{i}(x), \\
& \bar{v}(x)=\sum_{i=1}^{\infty} \bar{B}_{i} \bar{\psi}_{i}(x),
\end{aligned}
$$

where $\bar{A}_{i}=\sum_{k=1}^{i} \beta_{i k} F\left(x_{k}, \bar{u}_{k}\left(x_{k}\right), \bar{v}_{k}\left(p x_{k}\right)\right)$ and $\bar{B}_{i}=$ $\sum_{k=1}^{i} \beta_{i k} G\left(x_{k}, \bar{v}_{k}\left(x_{k}\right), \bar{u}_{k}\left(q x_{k}\right)\right)$.

In the proof of the convergence in Theorem 15 we only use $\left\|u_{n}\right\| \leq C,\left\|v_{n}\right\| \leq D$; thus we obtain the following corollary.

Corollary 16. Suppose $\left\|u_{n}\right\|$ and $\left\|v_{n}\right\|$ are bounded; then the iterative sequence (35) is convergent to the exact solution of (2).

Theorem 17. Assume $u(x)$ and $v(x)$ are the solutions of (2), $r_{n}^{1}=\left\|u(x)-u_{n}(x)\right\|_{W_{2}}$ and $r_{n}^{2}=\left\|v(x)-v_{n}(x)\right\|_{W_{2}}$ are the approximate errors of $u_{n}(x), v_{n}(x)$, where $u_{n}(x)$ and $v_{n}(x)$ are given by (35). Then the errors $r_{n}^{1}, r_{n}^{2}$ are monotone decreasing in the sense of $\|\cdot\|_{W_{2}}$.

Proof. From (35), and (79), it follows that

$$
\begin{aligned}
\left\|r_{n}^{1}(x)\right\|_{W_{2}}^{2} & =\left\|\sum_{i=n+1}^{\infty} \bar{A}_{i} \bar{\psi}_{i}(x)\right\|_{W_{2}}^{2} \\
& =\sum_{i=n+1}^{\infty}\left(\bar{A}_{i}\right)^{2} .
\end{aligned}
$$

In the same way, we obtain from (35), (80)

$$
\begin{aligned}
\left\|r_{n}^{2}(x)\right\|_{W_{2}}^{2} & =\left\|\sum_{i=n+1}^{\infty} \bar{B}_{i} \bar{\psi}_{i}(x)\right\|_{W_{2}}^{2} \\
& =\sum_{i=n+1}^{\infty}\left(\bar{B}_{i}\right)^{2} .
\end{aligned}
$$

Equations (81) and (82) show that the errors $r_{n}^{1}$ and $r_{n}^{2}$ are monotone decreasing in the sense of $\|\cdot\|_{W_{2}}$.

\section{Numerical Examples}

In order to demonstrate the efficiency of our algorithm for solving (2), we will present two numerical examples in the reproducing kernel space $W_{2}[0,+\infty)$. Let $n$ be the number of discrete points in $[0,+\infty)$. Denote $E_{n}^{u}(x) \stackrel{\text { def }}{=}$ $\left|\left(u(x)-u_{n}(x)\right) / u(x)\right|, E_{n}^{v}(x) \stackrel{\text { def }}{=}\left|\left(v(x)-v_{n}(x)\right) / v(x)\right|$. All computations are performed by the Mathematica 5.0 software package. Results obtained by the method are compared with the exact solution of each example and are found to be in good agreement with each other.

Example 18. In this example we consider the problem

$$
\begin{aligned}
& u^{\prime}(x)=\frac{3}{1+u(x)^{2}}+2 x \cos \left(v\left(\frac{1}{3} x\right)\right)+f(x), \\
& v^{\prime}(x)=2 e^{-v(x)}+4 x^{3} \sin \left(u\left(\frac{1}{2} x\right)\right)+g(x), \\
& u(0)=0, \quad v(0)=0,
\end{aligned}
$$

with exact solution $u(x)=x e^{-x}, v(x)=\sin x$, and $f(x)=e^{-x}-e^{-x} x-\left(3 /\left(1+e^{-2} x^{2}\right)\right)-2 x \cos (\sin (x / 3))$, $g(x)=-2 e^{-\sin x}+\cos x-4 x^{3} \sin \left((1 / 2) x e^{-x / 2}\right)$. Applying the presented method in Section 3, we calculate the approximate solution $u_{50}(x)$ and $v_{50}(x)$ in $[0,1]$ as follows.

Step 1. By the method of the appendix, the corresponding reproducing kernel functions can be obtained.

Step 2. Choosing a dense subset in $[0,1]$, then we get the orthogonalization coefficients $\beta_{i k}$.

Step 3. According to (10), we can get the normal orthogonal systems $\left\{\overline{\psi_{i}}(x)\right\}_{i=1}^{50}$.

Step 4. Selecting the initial value $u_{0}(x)=v_{0}(x)=0$, we obtain $u_{1}(x), u_{2}(x), \ldots, u_{50}(x)$ and $v_{1}(x), v_{2}(x), \ldots, v_{50}(x)$ by (19) developed in the paper.

The graphs of the superimposed image emerge in Figure 1. At the same time, we have computed the approximate solutions $u_{n}(x)$ and $v_{n}(x)(n=300,900)$ in $[0,3]$ and also calculated the relative errors $E_{n}^{u}$ and $E_{n}^{v}$ in Table 1 . The root mean square errors (RMSE) about $u(x)$ with $u_{n}$ and $v(x)$ with $v_{n}$ are shown in Table 2.

Example 19. Considering equations

$$
\begin{aligned}
& u^{\prime}(x)=3 \cos (u(x))+2 x \sin \left(v\left(\frac{1}{5} x\right)\right)+f(x), \\
& v^{\prime}(x)=2 \sin (v(x))+4 x^{3} \cos \left(u\left(\frac{1}{3} x\right)\right)+g(x), \\
& u(0)=0, \quad v(0)=0 .
\end{aligned}
$$




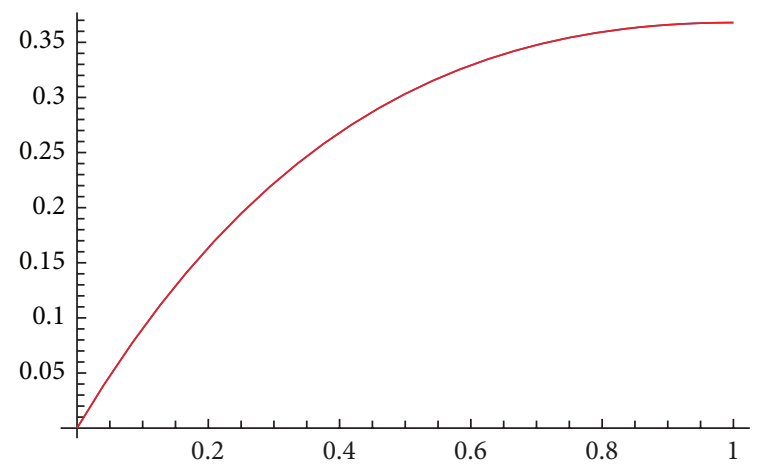

(a)

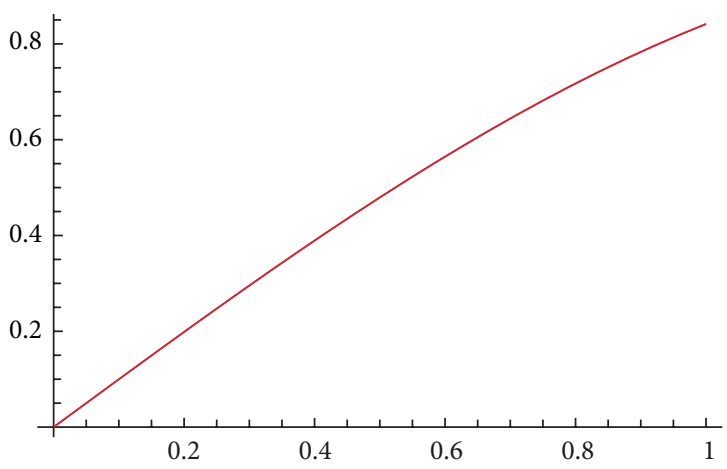

(b)

FIGURE 1: The left is the superimposed image of $u(x)$ with $u_{50}(x)$ in $[0,1]$. The right is the superimposed image of $v(x)$ with $v_{50}(x)$ in $[0,1]$.

TABLE 1: Relative errors in [0,3] for Example 18.

\begin{tabular}{lcccc}
\hline Node & $E_{300}^{u}$ & $E_{300}^{v}$ & $E_{900}^{u}$ & $E_{300}^{v}$ \\
\hline 0.3 & $4.97833 e-5$ & $8.4527 e-5$ & $5.53163 e-6$ & $9.39806 e-6$ \\
0.6 & $4.43080 e-5$ & $7.3330 e-5$ & $4.92288 e-6$ & $8.15664 e-6$ \\
0.9 & $3.86801 e-5$ & $6.6731 e-5$ & $4.29733 e-6$ & $7.42512 e-6$ \\
1.2 & $3.41875 e-5$ & $6.1845 e-5$ & $3.79797 e-6$ & $6.88400 e-6$ \\
1.5 & $3.09088 e-5$ & $5.7095 e-5$ & $3.43349 e-6$ & $6.35872 e-6$ \\
1.8 & $2.87391 e-5$ & $5.1303 e-5$ & $3.19221 e-6$ & $5.71898 e-6$ \\
2.1 & $2.75649 e-5$ & $4.3153 e-5$ & $3.06149 e-6$ & $4.81984 e-6$ \\
2.4 & $2.7288 e-5$ & $3.0422 e-5$ & $3.03034 e-6$ & $3.41736 e-6$ \\
2.7 & $2.7829 e-5$ & $6.7566 e-5$ & $3.09008 e-6$ & $8.12936 e-7$ \\
3.0 & $2.90670 e-5$ & $7.5017 e-5$ & $3.23206 e-6$ & $8.72219 e-6$ \\
\hline
\end{tabular}

TABLE 2: The RMS errors in [0,3] for Example 18.

\begin{tabular}{ccc}
\hline$n$ & $\sqrt{\sum_{i=1}^{n}\left(u_{n}\left(x_{i}\right)-u\left(x_{i}\right)\right)^{2} / n}$ & $\sqrt{\sum_{i=1}^{n}\left(v_{n}\left(x_{i}\right)-v\left(x_{i}\right)\right)^{2} / n}$ \\
\hline 300 & $9.95194 e-6$ & $4.00007 e-5$ \\
900 & $1.10551 e-6$ & $4.45554 e-6$ \\
\hline
\end{tabular}

The true solutions are $u(x)=5 x, v(x)=4 \tan (x), f(x)=$ $5-3 \cos (5 x)-2 x \sin (4 \tan (1 / 5)), g(x)=-4 x^{3} \cos (5 x / 3)+$ $4 \sec x^{2}-2 \sin (4 \tan x)$. The numerical results are given in Figure 2 and Tables 3 and 4 . The figures and tables illustrate that the method given in the paper is efficient.

\section{Conclusion}

In this paper, RKHSM has been successfully applied to find the solutions of systems of nonlinear IDDEs with proportional delays. The efficiency and accuracy of the proposed decomposition method were demonstrated by two test problems. It is concluded from above tables and figures that the RKHSM is an accurate and efficient method to solve IDDEs with proportional delays. Moreover, the method is also effective for solving some nonlinear initial-boundary value problems and nonlocal boundary value problems.
TABLE 3: Relative errors in [0,4] for Example 19.

\begin{tabular}{lcccc}
\hline Node & $E_{800}^{u}$ & $E_{800}^{v}$ & $E_{1200}^{u}$ & $E_{1200}^{u}$ \\
\hline 0.4 & $8.06470 e-5$ & $4.94886 e-5$ & $3.58334 e-5$ & $2.20033 e-5$ \\
0.8 & $1.07473 e-4$ & $5.27211 e-5$ & $4.77632 e-5$ & $2.34475 e-5$ \\
1.2 & $2.56877 e-4$ & $5.32904 e-5$ & $1.14199 e-4$ & $2.37040 e-5$ \\
1.6 & $1.19110 e-4$ & $5.01608 e-5$ & $5.29170 e-5$ & $2.23098 e-5$ \\
2.0 & $8.56122 e-5$ & $4.35915 e-5$ & $3.80383 e-5$ & $1.93810 e-5$ \\
2.4 & $2.27843 e-4$ & $3.52666 e-5$ & $1.01280 e-4$ & $1.56705 e-5$ \\
2.8 & $1.38237 e-4$ & $2.74018 e-5$ & $6.13912 e-5$ & $1.21676 e-5$ \\
3.2 & $7.26035 e-5$ & $2.15572 e-5$ & $3.22429 e-5$ & $9.56745 e-6$ \\
3.6 & $1.86684 e-5$ & $1.80587 e-5$ & $8.29627 e-5$ & $8.01449 e-6$ \\
4.0 & $1.50042 e-4$ & $1.64230 e-5$ & $6.66348 e-5$ & $7.14957 e-6$ \\
\hline
\end{tabular}

TABLE 4: The RMS errors in [0,4] for Example 19.

\begin{tabular}{ccc}
\hline$n$ & $\sqrt{\sum_{i=1}^{n}\left(u_{n}\left(x_{i}\right)-u\left(x_{i}\right)\right)^{2} / n}$ & $\sqrt{\sum_{i=1}^{n}\left(v_{n}\left(x_{i}\right)-v\left(x_{i}\right)\right)^{2} / n}$ \\
\hline 800 & $1.87807 e-3$ & $7.85856 e-5$ \\
1200 & $8.34276 e-4$ & $3.48826 e-5$ \\
\hline
\end{tabular}

\section{Appendix}

\section{The Reproducing Kernel Space $W_{2}[0, m]$}

$W_{2}[0, m]$ is defined as $W_{2}[0, m]=\left\{u(x) \mid u^{\prime}\right.$ are absolutely continuous real value functions, $\left.u^{\prime \prime} \in L^{2}[0, m], u(0)=0\right\}$. The inner product in $W_{2}[0, m]$ is given by

$$
\langle u(y), v(y)\rangle_{W_{2}}=\int_{0}^{m}\left(4 u v+5 u^{\prime} v^{\prime}+u^{\prime \prime} v^{\prime \prime}\right) d y
$$

where $u, v \in W_{2}[0, m]$ and the norm $\|u\|_{W_{2}}$ is denoted by $\|u\|_{W_{2}}=\sqrt{\langle u, u\rangle_{W_{2}}}$.

Theorem A.1. The space $W_{2}[0, m]$ is a reproducing kernel space; that is, for any $u(y) \in W_{2}[0, m]$ and each fixed $x \in$ $[0, m]$, there exists $R_{x}(y) \in W_{2}[0, m], y \in[0, m]$, such that 


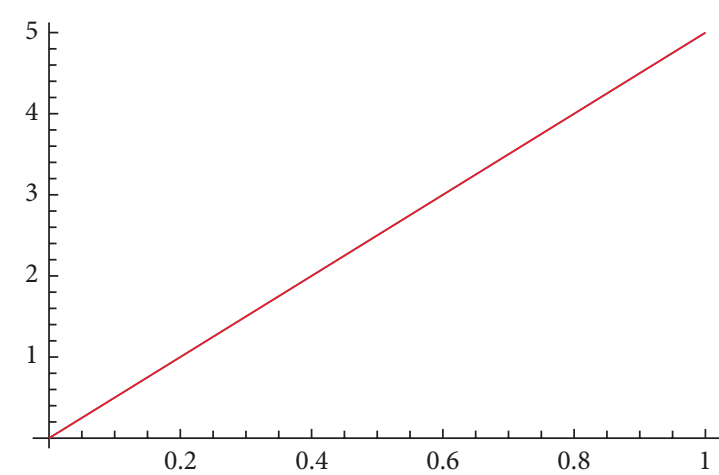

(a)

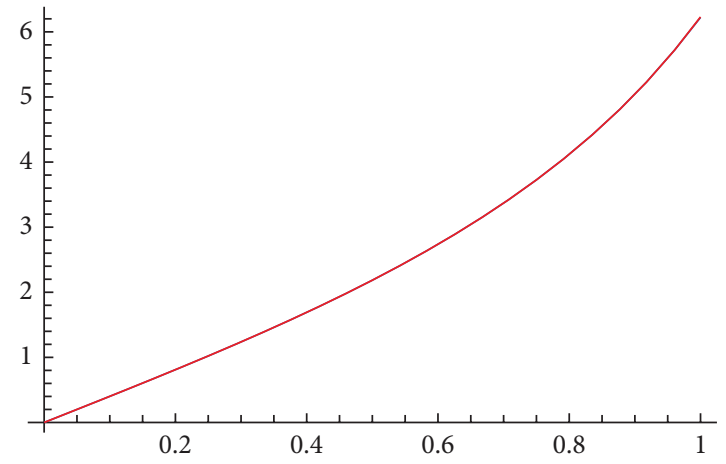

(b)

FIGURE 2: The left is the superimposed image of $u(x)$ with $u_{50}(x)$ in $[0,1]$. The right is the superimposed image of $v(x)$ with $v_{50}(x)$ in $[0,1]$.

$\left\langle u(y), R_{x}(y)\right\rangle_{W_{2}[0, m]}=u(x)$. The reproducing kernel $R_{x}(y)$ can be denoted by

$$
R_{x}(y)= \begin{cases}c_{1} e^{y}+c_{2} e^{-y}+c_{3} e^{2 y}+c_{4} e^{-2 y}, & y \leq x \\ d_{1} e^{y}+d_{2} e^{-y}+d_{3} e^{2 y}+d_{4} e^{-2 y}, & y>x\end{cases}
$$

Proof. Applying to the integrations by parts for (A.1), we have

$$
\begin{aligned}
& \left\langle u(y), R_{x}(y)\right\rangle_{W_{2}[0, m]} \\
& =\int_{0}^{m} u(y)\left[4 R_{x}(y)-5 R_{x}^{\prime \prime}(y)+R_{x}^{(4)}(y)\right] d y \\
& \quad+\left.5 u(y) R_{x}^{\prime}(y)\right|_{0} ^{m}+\left.u^{\prime}(y) R_{x}^{\prime \prime}(y)\right|_{0} ^{m} \\
& \quad-\left.u(y) R_{x}^{(3)}(y)\right|_{0} ^{m} .
\end{aligned}
$$

Since $R_{x}(y) \in W_{2}[0, m]$, it follows that

$$
R_{x}(0)=0 .
$$

For $u(y) \in W_{2}[0, m]$, thus, $u(0)=0$.

Suppose that $R_{x}(y)$ satisfies the following generalized differential equations:

$$
\begin{gathered}
4 R_{x}(y)-5 R_{x}^{\prime \prime}(y)+R_{x}^{(4)}(y)=\delta(y-x), \\
5 R_{x}^{\prime}(m)-R_{x}^{(3)}(m)=0, \\
R_{x}^{\prime \prime}(m)=0 \\
R_{x}^{\prime \prime}(0)=0 .
\end{gathered}
$$

Then $\left\langle u(y), R_{x}(y)\right\rangle_{W_{2}[0, m]}=\int_{0}^{m} u(y) \delta(y-x) d y=u(x)$. Hence, $R_{x}(y)$ is the reproducing kernel of space $W_{2}[0, m]$.

In the following, we will get the expression of the reproducing kernel $R_{x}(y)$.
The characteristic equation of $R_{x}(y)-5 R_{x}^{\prime \prime}(y)+R_{x}^{(4)}(y)=$ $\delta(y-x)$ is given by $\lambda^{4}-5 \lambda^{2}+4=0$, and the characteristic roots are $\lambda_{1,2}= \pm 1, \lambda_{3,4}= \pm 2$.

We denote $R_{x}(y)$ by

$$
R_{x}(y)= \begin{cases}c_{1} e^{y}+c_{2} e^{-y}+c_{3} e^{2 y}+c_{4} e^{-2 y}, & y \leq x, \\ d_{1} e^{y}+\mathrm{d}_{2} e^{-y}+d_{3} e^{2 y}+d_{4} e^{-2 y}, & y>x .\end{cases}
$$

By the definition of space $W_{2}[0, m]$, coefficients $c_{1}, \ldots, c_{4}$, $d_{1}, \ldots, d_{4}$ satisfy

$$
\begin{gathered}
R_{x}^{(k)}(x+0)=R_{x}^{(k)}(x-0) \quad(k=0,1,2), \\
R_{x}^{(3)}(m)-5 R_{x}^{\prime}(m)=0 \\
R_{x}^{(3)}(x+0)-R_{x}^{(3)}(x-0)=1 \\
R_{x}^{\prime \prime}(m)=0 \\
R_{x}^{\prime \prime}(0)=0 \\
R_{x}(0)=0
\end{gathered}
$$

from which, the unknown coefficients of (A.6) can be obtained:

$$
\begin{aligned}
& c_{1}= \\
& \frac{e^{-2 x}\left(4 e^{3 m}-7 e^{3 x}-9 e^{2 m+x}+7 e^{6 m+x}+9 e^{4 m+3 x}-4 e^{3 m+4 x}\right)}{6\left(-7-9 e^{2 m}+9 e^{4 m}+7 e^{6 m}\right)}
\end{aligned}
$$

$c_{2}=$

$$
\frac{e^{-2 x}\left(-4 e^{3 m}+7 e^{3 x}+9 e^{2 m+x}-7 e^{6 m+x}-9 e^{4 m+3 x}+4 e^{3 m+4 x}\right)}{6\left(-7-9 e^{2 m}+9 e^{4 m}+7 e^{6 m}\right)}
$$


$c_{3}=$

$$
\frac{e^{-2 x}\left(-9 e^{4 m}-7 e^{6 m}+7 e^{4 x}+8 e^{3 m+x}-8 e^{3(m+x)}+9 e^{2 m+4 x}\right)}{12\left(-7-9 e^{2 m}+9 e^{4 m}+7 e^{6 m}\right)}
$$

$c_{4}=$

$$
\frac{-e^{-2 x}\left(-9 e^{4 m}-7 e^{6 m}+7 e^{4 x}+8 e^{3 m+x}-8 e^{3(m+x)}+9 e^{2 m+4 x}\right)}{12\left(-7-9 e^{2 m}+9 e^{4 m}+7 e^{6 m}\right)}
$$

$d_{1}=$

$$
-\frac{e^{-2 x}\left(-1+e^{2 x}\right)\left(4 e^{3 m}+7 e^{x}-9 e^{4 m+x}+4 e^{3 m+2 x}\right)}{6\left(-7-9 e^{2 m}+9 e^{4 m}+7 e^{6 m}\right)}
$$

$d_{2}=$

$$
\frac{e^{2 m-2 x}\left(-1+e^{2 x}\right)\left(4 e^{m}+7 e^{4 m+x}-9 e^{x}+4 e^{m+2 x}\right)}{6\left(-7-9 e^{2 m}+9 e^{4 m}+7 e^{6 m}\right)}
$$

$d_{3}=$

$$
\frac{e^{-2 x}\left(-1+e^{2 x}\right)\left(7+9 e^{2 m}+7 e^{2 x}+9 e^{2(m+x)}-8 e^{3 m+x}\right)}{12\left(-7-9 e^{2 m}+9 e^{4 m}+7 e^{6 m}\right)}
$$

$d_{4}=$

$$
-\frac{e^{3 m-2 x}\left(-1+e^{2 x}\right)\left(9 e^{m}+7 e^{3 m}+9 e^{m+2 x}-8 e^{x}+7 e^{3 m+2 x}\right)}{12\left(-7-9 e^{2 m}+9 e^{4 m}+7 e^{6 m}\right)} .
$$

\section{Acknowledgments}

This research is supported by the National Natural Science Foundation of China (61071181), the Educational Department Scientific Technology Program of Heilongjiang Province (12531180, 12512133, and 12521148), and the Academic Foundation for Youth of Harbin Normal University (KGB201226).

\section{References}

[1] K. L. Cooke, "Functional-differential equations: some models and perturbation problems," in International Symposium on Differential Equations and Dynamical Systems, Held in Puerto Rico, J. Hale and J. LaSalle, Eds., pp. 167-183, Academic Press, New York, NY, USA, 1967.

[2] E. Fečkan, "On certain type of functional differential equations," Mathematica Slovaca, vol. 43, no. 1, pp. 39-43, 1994.

[3] L. J. Grimm, "Existence and continuous dependence for a class of nonlinear neutral-differential equations," Proceedings of the American Mathematical Society, vol. 29, no. 3, pp. 467-473, 1971.

[4] R. D. Driver, "A two-body problem of classical electrodynamics: the one-dimensional case," Annals of Physics, vol. 21, no. 1, pp. 122-142, 1963.

[5] R. J. Oberg, "On the local existence of solutions of certain functional-differential equations," Proceedings of the American Mathematical Society, vol. 20, pp. 295-302, 1969.
[6] Z. Jackiewicz, "Existence and uniqueness of solutions of neutral delay-differential equations with state dependent delays," Funkcialaj Ekvacioj, vol. 30, no. 1, pp. 9-17, 1987.

[7] J. G. Si and X. P. Wang, "Analytic solutions of a secondorder iterative functional differential equation," Computers \& Mathematics with Applications, vol. 43, no. 1-2, pp. 81-90, 2002.

[8] R. D. Nussbaum, "Existence and uniqueness theorems for some functional differential equations of neutral type," Journal of Differential Equations, vol. 11, no. 3, pp. 607-623, 1972.

[9] Y. Kuang and A. Feldstein, "Monotonic and oscillatory solutions of a linear neutral delay equation with infinite lag," SIAM Journal on Mathematical Analysis, vol. 21, no. 6, pp. 1633-1641, 1990.

[10] A. Iserles and Y. Liu, "On functional-differential equations with proportional delays," Tech. Rep. DAMTP, 1993/NA3, Cambridge University, 1993.

[11] Y. K. Liu, "Stability analysis of $\theta$-methods for neutral functionaldifferential equations," Numerische Mathematik, vol. 70, no. 4, pp. 473-485, 1995.

[12] E. Ishiwata and Y. Muroya, "Rational approximation method for delay differential equations with proportional delay," Applied Mathematics and Computation, vol. 187, no. 2, pp. 741-747, 2007.

[13] E. Ishiwata, Y. Muroya, and H. Brunner, "A super-attainable order in collocation methods for differential equations with proportional delay," Applied Mathematics and Computation, vol. 198, no. 1, pp. 227-236, 2008.

[14] P. Hu, C. Huang, and S. Wu, "Asymptotic stability of linear multistep methods for nonlinear neutral delay differential equations," Applied Mathematics and Computation, vol. 211, no. 1, pp. 95-101, 2009.

[15] W. Wang, Y. Zhang, and S. Li, "Stability of continuous RungeKutta-type methods for nonlinear neutral delay-differential equations," Applied Mathematical Modelling, vol. 33, no. 8, pp. 3319-3329, 2009.

[16] W. S. Wang and S. F. Li, "On the one-leg $\theta$-methods for solving nonlinear neutral functional differential equations," Applied Mathematics and Computation, vol. 193, no. 1, pp. 285-301, 2007.

[17] W. Wang, T. Qin, and S. Li, "Stability of one-leg $\theta$-methods for nonlinear neutral differential equations with proportional delay," Applied Mathematics and Computation, vol. 213, no. 1, pp. 177-183, 2009.

[18] X. Chen and L. Wang, "The variational iteration method for solving a neutral functional-differential equation with proportional delays," Computers \& Mathematics with Applications, vol. 59, no. 8, pp. 2696-2702, 2010.

[19] J. Biazar and B. Ghanbari, "The homotopy perturbation method for solving neutral functional differential equations with proportional delays," Journal of King Saud University, vol. 24, no. 1, pp. 33-37, 2012.

[20] M. De la Sen, "Stability of impulsive time-varying systems and compactness of the operators mapping the input space into the state and output spaces," Journal of Mathematical Analysis and Applications, vol. 321, no. 2, pp. 621-650, 2006.

[21] M. De la Sen, R. P. Agarwal, A. Ibeas, and S. Alonso-Quesada, "On a generalized time-varying SEIR epidemic model with mixed point and distributed time-varying delays and combined regular and impulsive vaccination controls," Advances in Difference Equations, vol. 2010, Article ID 281612, 42 pages, 2010.

[22] E. Fridman and Y. Orlov, "Exponential stability of linear distributed parameter systems with time-varying delays," Automatica, vol. 45, no. 1, pp. 194-201, 2009. 
[23] X. Lü and M. Cui, "Analytic solutions to a class of nonlinear infinite-delay-differential equations," Journal of Mathematical Analysis and Applications, vol. 343, no. 2, pp. 724-732, 2008.

[24] C. Zhang and G. Sun, "Nonlinear stability of Runge-Kutta methods applied to infinite-delay-differential equations," Mathematical and Computer Modelling, vol. 39, no. 4-5, pp. 495-503, 2004.

[25] X. Lv and Y. Gao, "The RKHSM for solving neutral functionaldifferential equation with proportional delays," Mathematical Methods in the Applied Sciences, vol. 36, no. 6, pp. 642-649.

[26] C. Minggen and D. Zhongxing, "Solution to the definite solution problem of differential equations in space $W_{2}^{1}[0,1]$," Advance in Mathematics, vol. 17, pp. 327-328, 1986.

[27] C. 1. Li and M. G. Cui, "The exact solution for solving a class nonlinear operator equations in the reproducing kernel space," Applied Mathematics and Computation, vol. 143, no. 2-3, pp. 393-399, 2003. 


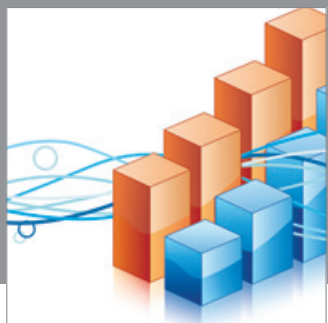

Advances in

Operations Research

mansans

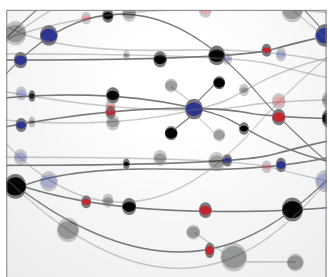

The Scientific World Journal
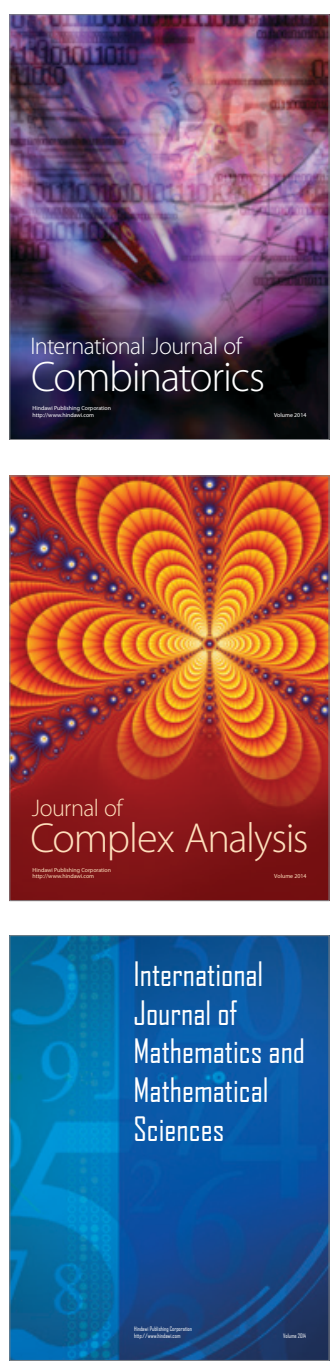
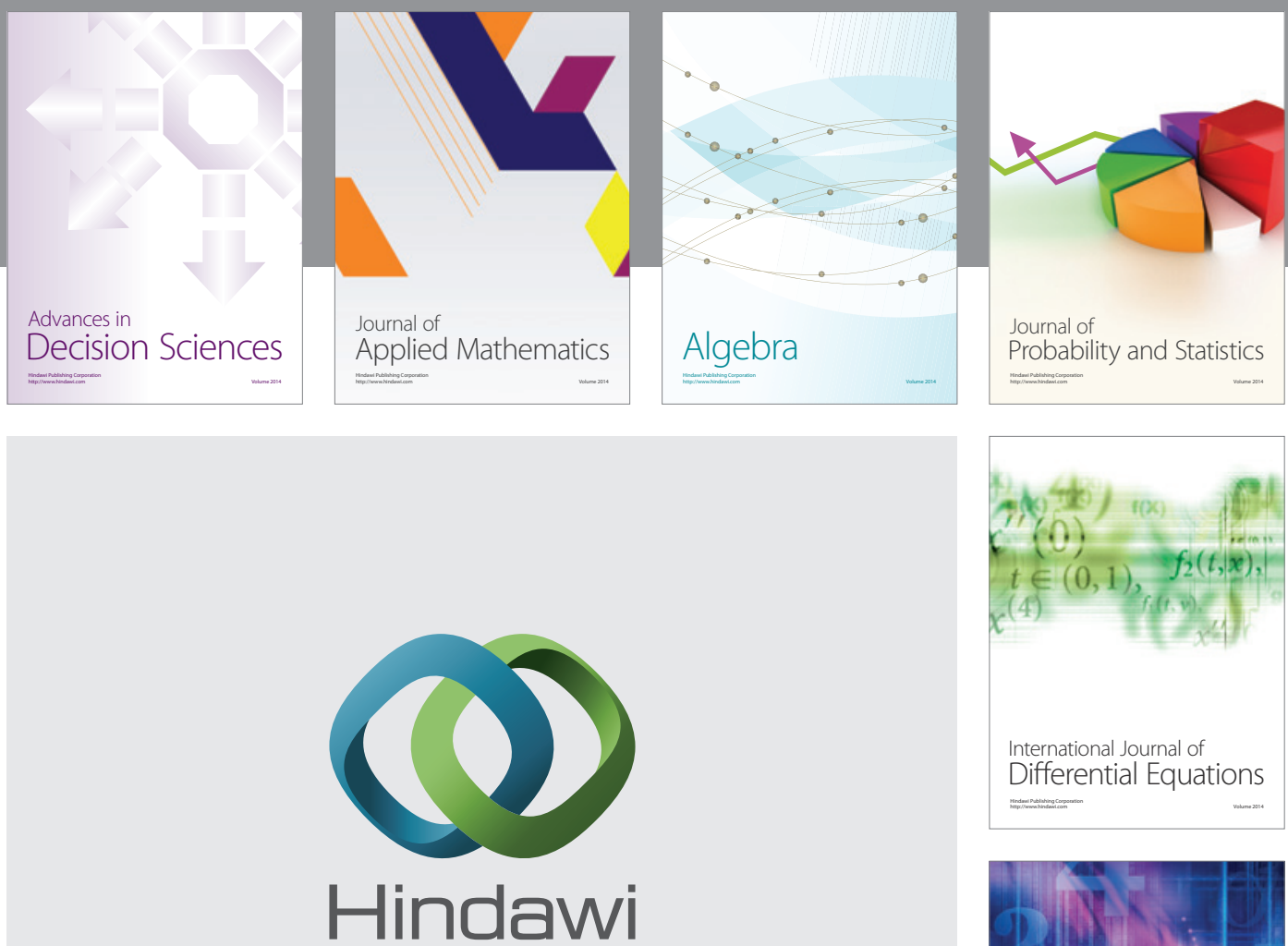

Submit your manuscripts at http://www.hindawi.com
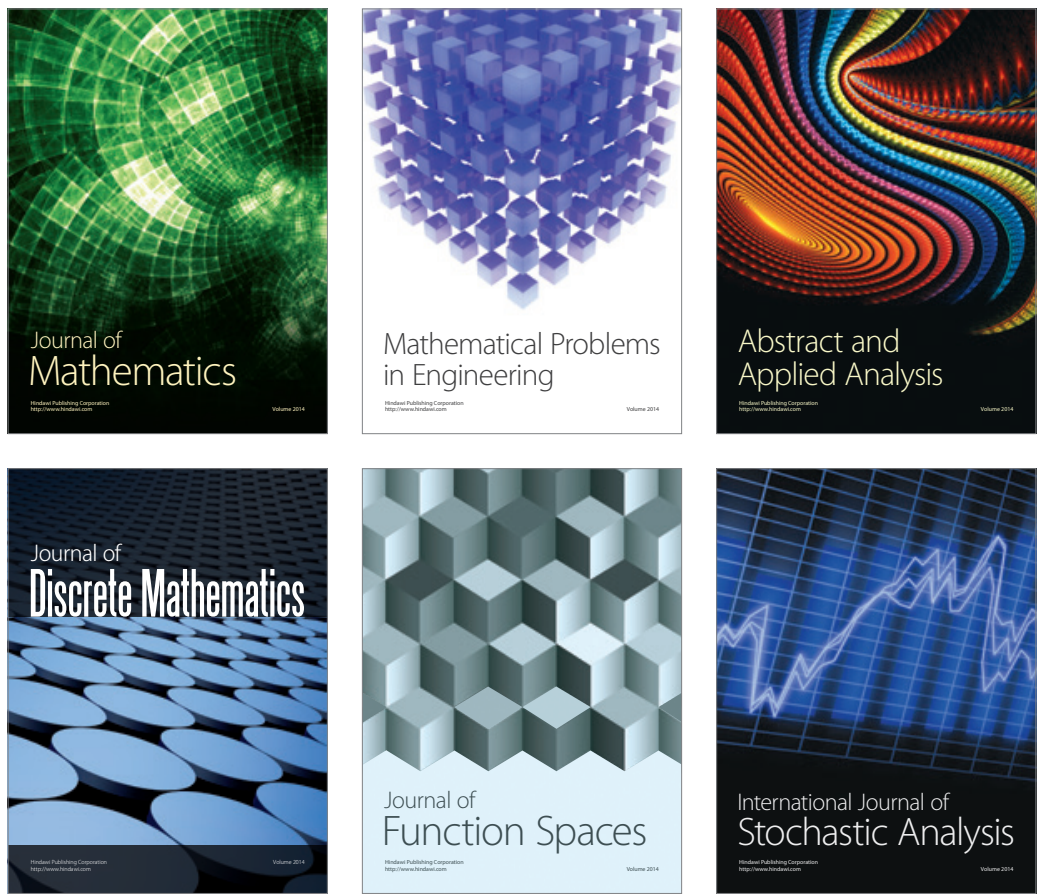

Journal of

Function Spaces

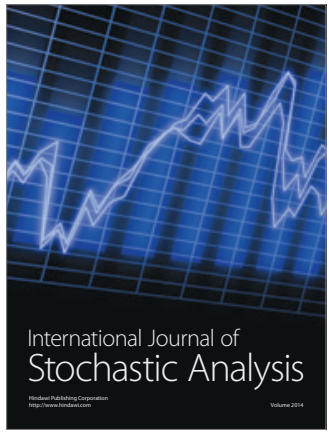

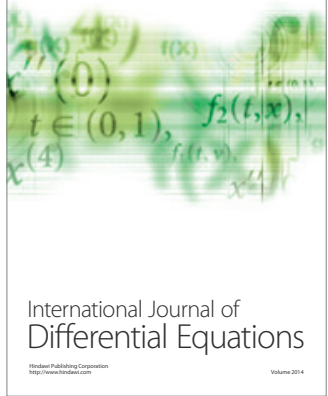
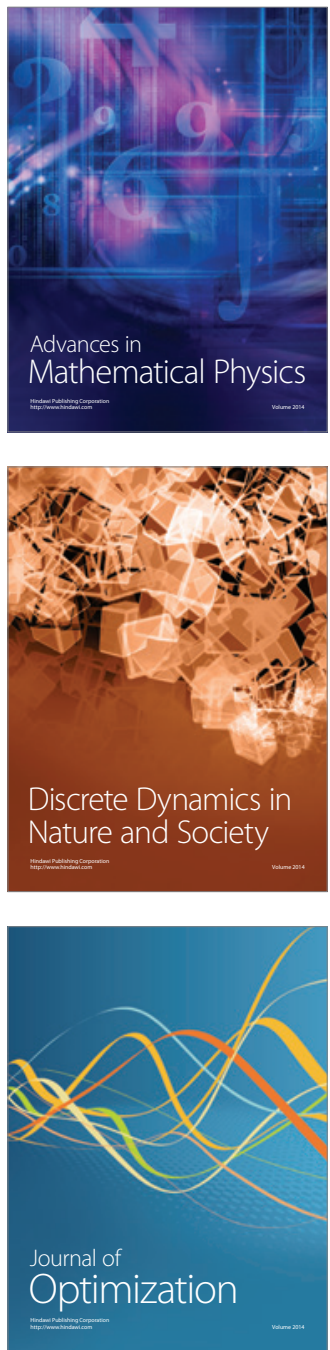